\title{
23. GRAIN SIZE ANALYSES
}

Robert E. Boyce, Scripps Institution of Oceanography, La Jolla, California

Aboard the Glomar Challenger 10-cc grain size samples were selected from each prominent lithology or every 1.5 meters where the lithology appeared uniform. The sand, silt, and clay-size percentages were determined and are listed in Table 1.

The sand, silt, and clay-size fractions were determined by standard sieving and pipette analysis. The sediment sample was dried and then dispersed in calgon solution. If the sediment failed to disaggregate in calgon, it was dispersed in hydrogen peroxide. The sand-size fraction was separated by a 62.5 micron sieve with the fines being processed via standard pipette analysis following Stokes settling velocity equation (Krumbein and Pettijohn, 1938, p. 95-96) which is discussed in Volume 9 of the Initial Reports of the Deep Sea Drilling Project. Step by step procedures are in Volume 4. When using splits of the same sample and having different operators over a long period of time the sand, silt, and clay-size fractions are reproducible within \pm 2.5 per cent (absolute).

Grain diameters can be expressed in phi units or microns, phi unit being the grain diameter in $\mathrm{mm}$ expressed as the negative log to the base 2 . These units and parameters are discussed in detail by Inman (1952). Sediment classification is after Shepard (1954) based on the Wentworth (1922) scale. The ternary diagram of Shepard's sediment classification is in Volume 9 of the Initial Reports of the Deep Sea Drilling Project.
TABLE 1 - Continued

\begin{tabular}{|c|c|c|c|c|}
\hline $\begin{array}{l}\text { Core, Section } \\
\text { Interval } \\
\text { (cm) }\end{array}$ & $\begin{array}{c}\text { Sand } \\
(\%)\end{array}$ & $\begin{array}{l}\text { Silt } \\
(\%)\end{array}$ & $\begin{array}{c}\text { Clay } \\
(\%)\end{array}$ & Classification \\
\hline \multicolumn{5}{|l|}{ DSDP 86} \\
\hline $\begin{array}{l}1-1(19.0) \\
1-1(39.0) \\
1-3(19.0)\end{array}$ & $\begin{array}{r}14.2 \\
6.8 \\
6.1\end{array}$ & $\begin{array}{l}21.3 \\
17.1 \\
21.7\end{array}$ & $\begin{array}{l}64.6 \\
76.0 \\
72.2\end{array}$ & $\begin{array}{l}\text { Silty clay } \\
\text { Clay } \\
\text { Silty clay }\end{array}$ \\
\hline $2-1(74.0)$ & 4.9 & 21.7 & 73.5 & Silty clay \\
\hline $\begin{array}{l}3-2(8.0) \\
3-3(14.0) \\
3-4(20.0) \\
3-5(30.0) \\
3-6(31.0)\end{array}$ & $\begin{array}{l}8.0 \\
7.1 \\
9.4 \\
7.4 \\
5.2\end{array}$ & $\begin{array}{r}6.4 \\
25.2 \\
22.7 \\
20.9 \\
21.4\end{array}$ & $\begin{array}{l}72.7 \\
67.6 \\
67.9 \\
71.7 \\
73.5\end{array}$ & $\begin{array}{l}\text { Silty clay } \\
\text { Silty clay } \\
\text { Silty clay } \\
\text { Silty clay } \\
\text { Silty clay }\end{array}$ \\
\hline $\begin{array}{l}4-2(29.0) \\
4-3(19.0) \\
4-4(29.0) \\
4-5(15.0)\end{array}$ & $\begin{array}{l}4.6 \\
8.5 \\
3.7 \\
2.2\end{array}$ & $\begin{array}{l}21.1 \\
25.1 \\
23.9 \\
23.0\end{array}$ & $\begin{array}{l}74.3 \\
66.4 \\
72.4 \\
74.8\end{array}$ & $\begin{array}{l}\text { Silty clay } \\
\text { Silty clay } \\
\text { Silty clay } \\
\text { Silty clay }\end{array}$ \\
\hline $\begin{array}{l}5-1(110.0) \\
5-3(12.0) \\
5-4(19.0)\end{array}$ & $\begin{array}{l}2.5 \\
3.2 \\
2.8\end{array}$ & $\begin{array}{l}45.6 \\
45.5 \\
41.8\end{array}$ & $\begin{array}{l}51.8 \\
51.3 \\
55.5\end{array}$ & $\begin{array}{l}\text { Silty clay } \\
\text { Silty clay } \\
\text { Silty clay }\end{array}$ \\
\hline $\begin{array}{l}7-3(19.0) \\
7-4(19.0) \\
7-5(21.0)\end{array}$ & $\begin{array}{r}12.3 \\
15.9 \\
8.2\end{array}$ & $\begin{array}{l}43.3 \\
29.7 \\
39.9\end{array}$ & $\begin{array}{l}44.4 \\
54.4 \\
52.0\end{array}$ & $\begin{array}{l}\text { Silty clay } \\
\text { Silty clay } \\
\text { Silty clay }\end{array}$ \\
\hline $\begin{array}{l}8-1(92.0) \\
8-3(18.0) \\
8-4(19.0)\end{array}$ & $\begin{array}{r}11.2 \\
22.9 \\
3.4\end{array}$ & $\begin{array}{l}79.8 \\
48.3 \\
42.3\end{array}$ & $\begin{array}{r}9.0 \\
28.8 \\
54.3\end{array}$ & $\begin{array}{l}\text { Silt } \\
\text { Sand-silt-clay } \\
\text { Silty clay }\end{array}$ \\
\hline $9-2(20.0)$ & 2.3 & 46.3 & 51.4 & Silty clay \\
\hline
\end{tabular}

DSDP 87

$1-1(135.0)$

51.1

20.0

Sandy silt

\section{DSDP 88}

\begin{tabular}{lrrrl}
\hline $\begin{array}{c}\text { Core, Section } \\
\text { Interval } \\
(\mathrm{cm})\end{array}$ & $\begin{array}{c}\text { Sand } \\
(\%)\end{array}$ & $\begin{array}{r}\text { Silt } \\
(\%)\end{array}$ & $\begin{array}{r}\text { Clay } \\
(\%)\end{array}$ & Classification \\
\hline DSDP 85 & & & & \\
& & & & \\
1-1 (14.0) & 0.1 & 22.8 & 77.1 & Clay \\
1-2(10.0) & 1.0 & 28.9 & 70.1 & Silty clay \\
$1-3(29.0)$ & 5.9 & 30.7 & 63.4 & Silty clay \\
$1-4(13.0)$ & 2.9 & 30.9 & 66.2 & Silty clay \\
$1-4(88.0)$ & 0.1 & 26.0 & 74.0 & Silty clay \\
$1-6(10.0)$ & 0.1 & 24.5 & 75.5 & Clay \\
2-1 (4.0) & 4.7 & 27.8 & 67.4 & Silty clay \\
2-3(17.0) & 0.0 & 29.0 & 71.0 & Silty clay \\
3-1 (5.0) & 1.1 & 21.9 & 77.0 & Clay \\
3-3 (4.0) & 0.0 & 21.7 & 78.3 & Clay \\
3-4 (13.0) & 2.0 & 41.0 & 57.0 & Silty clay \\
3-6 (57.0) & 0.0 & 13.3 & 86.7 & Clay \\
4-1 (9.5) & 1.5 & 80.8 & 17.6 & Silt \\
$5-1(77.0)$ & 94.3 & 2.1 & 3.6 & Sand \\
$5-2(29.0)$ & 96.5 & 1.2 & 2.4 & Sand \\
\hline
\end{tabular}

$1-2(24.0)$

$1-3(9.0)$

1-4 (10.0)

2-2 (30.0)

2-3 (31.0)

2-4 (49.0)

$2-5(40.0)$

2-6 (20.0)

3-3 (74.0)

3-4 (10.0)

$3-5(17.0)$

3-3 $(80.0)$

4-3 (140.0)

4-5 (28.0)

5-5 (70.0)

5-6 (58.0)

DSDP 89

$\begin{array}{llll}6.9 & 15.0 & 78.1 & \text { Clay } \\ 3.7 & 16.4 & 79.9 & \text { Clay } \\ 3.5 & 17.1 & 79.4 & \text { Clay } \\ 6.1 & 17.1 & 76.8 & \text { Clay } \\ 2.4 & 16.7 & 80.9 & \text { Clay } \\ 5.1 & 18.6 & 76.4 & \text { Clay } \\ 5.8 & 30.9 & 63.3 & \text { Silty clay } \\ 3.5 & 18.0 & 78.5 & \text { Clay } \\ 5.8 & 18.3 & 75.9 & \text { Clay } \\ 3.2 & 24.4 & 72.3 & \text { Silty clay } \\ 3.5 & 21.4 & 75.1 & \text { Clay } \\ 2.7 & 24.4 & 72.9 & \text { Silty clay } \\ 2.7 & 18.4 & 78.9 & \text { Clay } \\ 1.0 & 12.9 & 86.1 & \text { Clay } \\ 1.1 & 16.0 & 82.8 & \text { Clay } \\ 0.7 & 15.7 & 83.6 & \text { Clay }\end{array}$

\begin{tabular}{lllll}
$1-2(5.0)$ & 3.2 & 14.4 & 82.4 & Clay \\
$1-3(19.0)$ & 2.2 & 10.5 & 87.3 & Clay \\
\hline
\end{tabular}


TABLE 1 - Continued

\begin{tabular}{lcccl}
\hline $\begin{array}{c}\text { Core, Section } \\
\text { Interval } \\
(\mathrm{cm})\end{array}$ & $\begin{array}{c}\text { Sand } \\
(\%)\end{array}$ & $\begin{array}{c}\text { Silt } \\
(\%)\end{array}$ & $\begin{array}{c}\text { Clay } \\
(\%)\end{array}$ & Classification \\
\hline $2-1(140.0)$ & 6.4 & 15.4 & 78.2 & Clay \\
$3-2(30.0)$ & 1.5 & 21.0 & 77.5 & Clay \\
$3-3(25.0)$ & 3.9 & 28.4 & 67.7 & Silty clay \\
$3-4(30.0)$ & 3.2 & 18.3 & 78.5 & Clay \\
$4-2(20.0)$ & 1.1 & 19.1 & 79.8 & Clay \\
$4-4(40.0)$ & 2.4 & 22.8 & 74.8 & Silty clay \\
4-5 (49.0) & 0.9 & 19.8 & 79.3 & Clay \\
4-6 (20.0) & 3.9 & 25.5 & 70.6 & Silty clay \\
5-1 (110.0) & 0.3 & 32.1 & 67.6 & Silty clay \\
$6-5(23.0)$ & 0.9 & 37.5 & 61.6 & Silty clay \\
$6-4(30.0)$ & 1.3 & 35.7 & 63.0 & Silty clay
\end{tabular}

\section{DSDP 90}

\begin{tabular}{lrrrl} 
1-2 (20.0) & 0.2 & 36.5 & 63.3 & Silty clay \\
$1-3(19.0)$ & 2.0 & 34.9 & 63.1 & Silty clay \\
$1-4(20.0)$ & 2.1 & 11.4 & 86.5 & Clay \\
$1-5(20.0)$ & 1.2 & 21.7 & 77.1 & Clay \\
$1-6(20.0)$ & 0.9 & 24.3 & 74.9 & Silty clay \\
$2-3(20.0)$ & 0.2 & 29.0 & 70.8 & Silty clay \\
2-4 (75.0) & 0.8 & 31.0 & 68.1 & Silty clay \\
3-2(20.0) & 1.1 & 13.5 & 85.4 & Clay \\
3-3(22.0) & 0.6 & 13.9 & 85.5 & Clay \\
$4-2(20.0)$ & 0.1 & 11.3 & 88.7 & Clay \\
$5-2(20.0)$ & 0.6 & 16.6 & 82.8 & Clay \\
$5-3(20.0)$ & 0.3 & 14.4 & 85.3 & Clay \\
$5-4(10.0)$ & 0.3 & 18.0 & 81.7 & Clay \\
6-2(20.0) & 0.1 & 53.1 & 46.8 & Clayey silt \\
$7-1(59.0)$ & 0.1 & 27.9 & 72.0 & Silty clay \\
$7-2(20.0)$ & 0.1 & 28.3 & 71.6 & Silty clay \\
$7-3(20.0)$ & 0.1 & 36.9 & 63.1 & Silty clay \\
$9-1(45.0)$ & 1.6 & 43.5 & 54.9 & Silty clay \\
$10-1(8.0)$ & 13.0 & 47.8 & 39.2 & Clayey silt \\
$13-3(84.0)$ & 78.6 & 14.6 & 6.8 & Sand \\
$13-4(35.0)$ & 79.7 & 13.5 & 6.7 & Sand \\
DSDP 91 & & & & \\
& & & & \\
$1-2(20.0)$ & 0.0 & 29.0 & 71.0 & Silty clay \\
$1-3(20.0)$ & 6.7 & 75.4 & 17.9 & Silt \\
$1-4(20.0)$ & 0.6 & 22.2 & 77.2 & Clay \\
$1-6(20.0)$ & 0.1 & 11.2 & 88.7 & Clay \\
$2-4(20.0)$ & 0.0 & 36.7 & 63.2 & Silty clay \\
$2-5(20.0)$ & 0.5 & 15.8 & 83.7 & Clay \\
$3-2(6.0)$ & 0.2 & 21.4 & 78.4 & Clay \\
$3-6(20.0)$ & 4.8 & 58.8 & 36.4 & Clayey silt \\
$4-2(20.0)$ & 1.1 & 41.8 & 57.1 & Silty clay \\
$4-3(20.0$ & 2.8 & 33.5 & 63.7 & Silty clay \\
$4-4(20.0)$ & 0.3 & 50.6 & 49.0 & Clayey silt \\
$4-5(20.0)$ & 0.0 & 36.8 & 63.2 & Silty clay \\
$4-6(10.0)$ & 0.3 & 15.7 & 84.0 & Clay \\
$5-2(8.0)$ & 0.4 & 32.6 & 67.0 & Silty clay \\
$5-3(10.0)$ & 0.0 & 27.5 & 72.4 & Silty clay \\
$5-4(15.0)$ & 0.0 & 45.7 & 54.3 & Silty clay \\
$5-6(5.0)$ & 0.0 & 29.8 & 70.2 & Silty clay \\
$6-2(10.0)$ & 0.0 & 21.2 & 78.8 & Clay \\
$6-3(20.0)$ & 0.0 & 22.0 & 78.0 & Clay \\
$6-4(20.0)$ & 0.0 & 26.5 & 73.5 & Silty clay \\
$6-5(5.0)$ & 0.0 & 41.0 & 59.0 & Silty clay \\
\hline & & & &
\end{tabular}

TABLE 1 - Continued

\begin{tabular}{|c|c|c|c|c|}
\hline $\begin{array}{l}\text { Core, Section } \\
\text { Interval } \\
\text { (cm) }\end{array}$ & $\begin{array}{c}\text { Sand } \\
(\%)\end{array}$ & $\begin{array}{l}\text { Silt } \\
(\%)\end{array}$ & $\begin{array}{c}\text { Clay } \\
(\%)\end{array}$ & Classification \\
\hline $7-1(70.0)$ & 26.4 & 66.6 & 6.9 & Sandy silt \\
\hline $7-2(30.0)$ & 28.2 & 65.5 & 6.3 & Sandy silt \\
\hline $7-3(10.0)$ & 54.9 & 41.2 & 3.9 & Silty sand \\
\hline $7-4(20.0)$ & 1.4 & 38.4 & 60.2 & Silty clay \\
\hline $7-5(20.0)$ & 0.5 & 25.1 & 74.4 & Silty clay \\
\hline 8-1 (20.0) & 47.9 & 34.2 & 17.9 & Silty sand \\
\hline $8-2(20.0)$ & 54.7 & 29.4 & & Silty sand \\
\hline $9-1(20.0)$ & 54.5 & 29.4 & 16.1 & Silty sand \\
\hline $9-2(20.0)$ & 53.0 & 36.7 & 10.4 & Silty sand \\
\hline $9-3(20.0)$ & 74.8 & 20.3 & 4.8 & Silty sand \\
\hline $9-4(20.0)$ & 57.9 & 23.4 & 18.7 & Silty sand \\
\hline $9-6(40.0)$ & 1.9 & 14.2 & 83.9 & Clay \\
\hline $10-2(20.0)$ & 18.3 & 51.6 & 30.1 & Clayey silt \\
\hline $10-4(20.0)$ & 21.2 & 62.4 & 16.4 & Sandy silt \\
\hline $11-2(20.0)$ & 77.6 & 17.4 & 4.9 & Sand \\
\hline $11-3(10.0)$ & 2.7 & 20.9 & 76.5 & Clay \\
\hline $11-4(20.0)$ & 0.0 & 16.4 & 83.6 & Clay \\
\hline $13-2(20.0)$ & 3.6 & 30.2 & 66.2 & Silty clay \\
\hline $13-3(20.0)$ & 1.4 & 18.3 & 80.3 & Clay \\
\hline $15-2(20.0)$ & 1.0 & 35.5 & 63.5 & Silty clay \\
\hline $15-3(20.0)$ & 42.4 & 9.9 & 47.7 & Sandy clay \\
\hline $16-2(80.0)$ & 0.9 & 27.0 & 72.1 & Silty clay \\
\hline $16-3(20.0)$ & 9.1 & 55.4 & 35.5 & Clayey silt \\
\hline $18-6(134.0)$ & 0.2 & 81.9 & 17.9 & Silt \\
\hline $20-2(20.0)$ & $\begin{array}{l}84.9 \\
74.9\end{array}$ & 10.6 & 4.5 & Sand \\
\hline $20-6(20.0)$ & & 14.5 & 10.6 & Silty sand \\
\hline $\begin{array}{l}22-1(20.0) \\
22-2(10.0)\end{array}$ & $\begin{array}{r}84.3 \\
2.1\end{array}$ & $\begin{array}{r}8.0 \\
25.2\end{array}$ & $\begin{array}{r}7.6 \\
72.7\end{array}$ & $\begin{array}{l}\text { Sand } \\
\text { Silty clay }\end{array}$ \\
\hline $\begin{array}{l}22-2(10.0) \\
25-1(100)\end{array}$ & & & & Sand \\
\hline $\begin{array}{l}25-1(110.0) \\
25-2(20.0)\end{array}$ & $\begin{array}{l}95.6 \\
96.1\end{array}$ & $\begin{array}{l}2.2 \\
2.1\end{array}$ & $\begin{array}{l}2.2 \\
1.7\end{array}$ & $\begin{array}{l}\text { Sand } \\
\text { Sand }\end{array}$ \\
\hline $\begin{array}{l}25-2(20.0) \\
25-3(40.0)\end{array}$ & 97.7 & 0.7 & 1.6 & Sand \\
\hline \multicolumn{5}{|l|}{ DSDP 92} \\
\hline $2-2(20.0)$ & 0.0 & 25.2 & 74.8 & Silty clay \\
\hline $2-3(20.0)$ & 0.1 & 27.0 & 72.9 & Silty clay \\
\hline $2-4(20.0)$ & 0.0 & 26.5 & 73.5 & Silty clay \\
\hline $2-5(20.0)$ & 0.1 & 26.1 & 73.8 & Silty clay \\
\hline $3-2(20.0)$ & 0.0 & 36.3 & 63.6 & Silty clay \\
\hline $3-3(20.0)$ & 0.1 & 28.1 & 71.9 & Silty clay \\
\hline $3-4(20.0)$ & 0.0 & 32.7 & 67.3 & Silty clay \\
\hline $3-5(20.0)$ & 0.0 & 31.6 & 68.3 & Silty clay \\
\hline $4-1(20.0)$ & 0.0 & 30.3 & 69.6 & Silty clay \\
\hline $4-3(20.0)$ & 0.0 & 28.6 & 71.4 & Silty clay \\
\hline $4-5(20.0)$ & 0.0 & 34.9 & 65.1 & Silty clay \\
\hline $5-2(20.0)$ & 0.1 & 26.9 & 73.1 & Silty clay \\
\hline $5-3(20.0)$ & 0.0 & 24.9 & 75.1 & Clay \\
\hline $5-4(22.0)$ & 0.1 & 24.5 & 75.3 & Clay \\
\hline $5-5(20.0)$ & 0.0 & 25.9 & 74.1 & Silty clay \\
\hline $5-6(20.0)$ & 0.0 & 24.8 & 75.2 & Clay \\
\hline $6-2(127.0)$ & 6.0 & 36.9 & 57.1 & Silty clay \\
\hline $8-1(103.0)$ & 0.1 & 33.1 & 66.8 & Silty clay \\
\hline \multicolumn{5}{|l|}{ DSDP 93} \\
\hline $1-2(20.0)$ & 0.4 & 36.9 & 62.7 & Silty clay \\
\hline \multicolumn{5}{|l|}{ DSDP 94} \\
\hline $1-2(20.0)$ & 4.6 & 29.4 & 66.0 & Silty clay \\
\hline
\end{tabular}


TABLE 1 - Continued

\begin{tabular}{|c|c|c|c|c|}
\hline $\begin{array}{l}\text { Core, Section } \\
\text { Interval } \\
\text { (cm) }\end{array}$ & $\begin{array}{c}\text { Sand } \\
(\%)\end{array}$ & $\begin{array}{l}\text { Silt } \\
(\%)\end{array}$ & $\begin{array}{c}\text { Clay } \\
(\%)\end{array}$ & Classification \\
\hline $\begin{array}{l}2-2(20.0) \\
2-3(20.0) \\
2-4(20.0) \\
2-5(20.0) \\
2-6(20.0)\end{array}$ & $\begin{array}{r}13.1 \\
9.3 \\
12.4 \\
9.2 \\
7.8\end{array}$ & $\begin{array}{l}29.1 \\
36.1 \\
28.0 \\
21.7 \\
22.3\end{array}$ & $\begin{array}{l}57.8 \\
54.6 \\
59.7 \\
69.0 \\
69.9\end{array}$ & $\begin{array}{l}\text { Silty clay } \\
\text { Silty clay } \\
\text { Silty clay } \\
\text { Silty clay } \\
\text { Silty clay }\end{array}$ \\
\hline $\begin{array}{l}3-2(20.0) \\
3-3(20.0) \\
3-4(20.0) \\
3-5(128.0) \\
3-6(20.0)\end{array}$ & $\begin{array}{r}10.0 \\
8.4 \\
9.9 \\
6.8 \\
9.9\end{array}$ & $\begin{array}{l}29.4 \\
30.9 \\
28.2 \\
32.5 \\
31.9\end{array}$ & $\begin{array}{l}60.6 \\
60.7 \\
61.9 \\
60.7 \\
58.2\end{array}$ & $\begin{array}{l}\text { Silty clay } \\
\text { Silty clay } \\
\text { Silty clay } \\
\text { Silty clay } \\
\text { Silty clay }\end{array}$ \\
\hline $\begin{array}{l}4-2(20.0) \\
4-3(20.0) \\
4-4(20.0) \\
4-5(20.0) \\
4-6(20.0)\end{array}$ & $\begin{array}{l}6.6 \\
4.8 \\
5.5 \\
4.9 \\
5.9\end{array}$ & $\begin{array}{l}23.0 \\
20.4 \\
22.5 \\
22.4 \\
26.6\end{array}$ & $\begin{array}{l}70.4 \\
74.8 \\
72.0 \\
72.6 \\
67.5\end{array}$ & $\begin{array}{l}\text { Silty clay } \\
\text { Silty clay } \\
\text { Silty clay } \\
\text { Silty clay } \\
\text { Silty clay }\end{array}$ \\
\hline $\begin{array}{l}5-2(20.0) \\
5-3(20.0) \\
5-4(20.0) \\
5-5(20.0) \\
5-6(20.0)\end{array}$ & $\begin{array}{l}5.4 \\
8.3 \\
4.8 \\
6.1 \\
6.2\end{array}$ & $\begin{array}{l}24.3 \\
26.2 \\
22.7 \\
27.2 \\
26.9\end{array}$ & $\begin{array}{l}70.4 \\
65.5 \\
72.4 \\
66.8 \\
66.9\end{array}$ & $\begin{array}{l}\text { Silty clay } \\
\text { Silty clay } \\
\text { Silty clay } \\
\text { Silty clay } \\
\text { Silty clay }\end{array}$ \\
\hline $\begin{array}{l}6-2(20.0) \\
6-4(20.0) \\
6-4(30.0) \\
6-5(20.0) \\
6-6(20.0)\end{array}$ & $\begin{array}{l}6.5 \\
1.5 \\
4.6 \\
3.9 \\
5.7\end{array}$ & $\begin{array}{l}31.1 \\
37.4 \\
27.1 \\
30.6 \\
27.4\end{array}$ & $\begin{array}{l}62.5 \\
61.1 \\
68.2 \\
65.5 \\
66.9\end{array}$ & $\begin{array}{l}\text { Silty clay } \\
\text { Silty clay } \\
\text { Silty clay } \\
\text { Silty clay } \\
\text { Silty clay }\end{array}$ \\
\hline $\begin{array}{l}7-3(20.0) \\
7-4(20.0) \\
7-5(20.0)\end{array}$ & $\begin{array}{l}2.4 \\
3.7 \\
3.9\end{array}$ & $\begin{array}{l}32.3 \\
29.1 \\
35.3\end{array}$ & $\begin{array}{l}65.3 \\
67.2 \\
60.8\end{array}$ & $\begin{array}{l}\text { Silty clay } \\
\text { Silty clay } \\
\text { Silty clay }\end{array}$ \\
\hline $\begin{array}{l}8-3(20.0) \\
8-4(20.0) \\
8-5(20.0)\end{array}$ & $\begin{array}{l}7.5 \\
2.5 \\
9.8\end{array}$ & $\begin{array}{l}34.0 \\
34.2 \\
44.3\end{array}$ & $\begin{array}{l}58.5 \\
63.3 \\
46.0\end{array}$ & $\begin{array}{l}\text { Silty clay } \\
\text { Silty clay } \\
\text { Silty clay }\end{array}$ \\
\hline $\begin{array}{l}9-2(20.0) \\
9-3(20.0) \\
9-4(20.0) \\
9-5(20.0) \\
9-6(20.0)\end{array}$ & $\begin{array}{l}13.3 \\
11.9 \\
12.3 \\
22.9 \\
12.4\end{array}$ & $\begin{array}{l}41.0 \\
43.0 \\
40.7 \\
42.0 \\
39.9\end{array}$ & $\begin{array}{l}45.6 \\
45.0 \\
47.0 \\
35.1 \\
47.7\end{array}$ & $\begin{array}{l}\text { Silty clay } \\
\text { Silty clay } \\
\text { Silty clay } \\
\text { Sand-silt-clay } \\
\text { Silty clay }\end{array}$ \\
\hline $\begin{array}{l}10-2(20.0) \\
10-3(20.0)\end{array}$ & $\begin{array}{l}2.5 \\
3.2\end{array}$ & $\begin{array}{l}41.6 \\
45.0\end{array}$ & $\begin{array}{l}55.9 \\
51.8\end{array}$ & $\begin{array}{l}\text { Silty clay } \\
\text { Silty clay }\end{array}$ \\
\hline $\begin{array}{l}11-2(20.0) \\
11-3(20.0) \\
11-4(20.0) \\
11-5(20.0) \\
11-6(20.0)\end{array}$ & $\begin{array}{l}4.3 \\
3.8 \\
4.2 \\
4.0 \\
4.0\end{array}$ & $\begin{array}{l}49.3 \\
47.6 \\
48.5 \\
47.6 \\
48.2\end{array}$ & $\begin{array}{l}46.4 \\
48.6 \\
47.3 \\
48.3 \\
47.8\end{array}$ & $\begin{array}{l}\text { Clayey silt } \\
\text { Silty clay } \\
\text { Clayey silt } \\
\text { Silty clay } \\
\text { Clayey silt }\end{array}$ \\
\hline $12-4(20.0)$ & 10.4 & 50.2 & 39.4 & Clayey silt \\
\hline $13-3(20.0)$ & 7.8 & 50.5 & 41.7 & Clayey silt \\
\hline $14-4(20.0)$ & 1.8 & 54.2 & 44.0 & Clayey silt \\
\hline $15-4(64.0)$ & 6.8 & 61.8 & 31.4 & Clayey silt \\
\hline $16-3(20.0)$ & 8.9 & 57.2 & 33.9 & Clayey silt \\
\hline $17-5(20.0)$ & 8.6 & 55.4 & 35.9 & Clayey silt \\
\hline $18-4(20.0)$ & 5.3 & 57.3 & 37.4 & Clayey silt \\
\hline $21-2(20.0)$ & 3.2 & 55.9 & 40.9 & Clayey silt \\
\hline \multicolumn{5}{|l|}{ DSDP 95} \\
\hline $1-1(110.0)$ & 19.9 & 25.3 & 54.8 & Silty clay \\
\hline $\begin{array}{l}2-1(20.0) \\
2-3(20.0) \\
2-6(20.0) \\
3-1(20.0) \\
3-2(110.0)\end{array}$ & $\begin{array}{r}9.6 \\
13.0 \\
16.8 \\
17.2 \\
8.5\end{array}$ & $\begin{array}{l}51.9 \\
45.6 \\
47.9 \\
39.4 \\
42.5\end{array}$ & $\begin{array}{l}38.5 \\
41.4 \\
35.3 \\
43.4 \\
49.0\end{array}$ & $\begin{array}{l}\text { Clayey silt } \\
\text { Clayey silt } \\
\text { Clayey silt } \\
\text { Silty clay } \\
\text { Silty clay }\end{array}$ \\
\hline
\end{tabular}

TABLE 1 - Continued

\begin{tabular}{|c|c|c|c|c|}
\hline $\begin{array}{l}\text { Core, Section } \\
\text { Interval } \\
\text { (cm) }\end{array}$ & $\begin{array}{c}\text { Sand } \\
(\%)\end{array}$ & $\begin{array}{l}\text { Silt } \\
(\%)\end{array}$ & $\begin{array}{c}\text { Clay } \\
(\%)\end{array}$ & Classification \\
\hline $\begin{array}{l}3-3(80.0) \\
3-4(40.0) \\
3-5(20.0) \\
3-6(20.0)\end{array}$ & $\begin{array}{r}8.9 \\
2.0 \\
7.1 \\
11.4\end{array}$ & $\begin{array}{l}41.3 \\
85.7 \\
43.2 \\
44.3\end{array}$ & $\begin{array}{l}49.7 \\
12.3 \\
49.7 \\
44.3\end{array}$ & $\begin{array}{l}\text { Silty clay } \\
\text { Silt } \\
\text { Silty clay } \\
\text { Clayey silt }\end{array}$ \\
\hline $\begin{array}{l}4-2(20.0) \\
4-3(20.0)\end{array}$ & $\begin{array}{r}7.0 \\
10.3\end{array}$ & $\begin{array}{l}50.6 \\
47.7\end{array}$ & $\begin{array}{l}42.4 \\
42.0\end{array}$ & $\begin{array}{l}\text { Clayey silt } \\
\text { Clayey silt }\end{array}$ \\
\hline $\begin{array}{l}5-1(20.0) \\
5-2(20.0) \\
5-3(20.0) \\
5-4(20.0)\end{array}$ & $\begin{array}{r}12.9 \\
6.0 \\
12.7 \\
11.9\end{array}$ & $\begin{array}{l}53.6 \\
60.3 \\
55.2 \\
55.8\end{array}$ & $\begin{array}{l}33.5 \\
33.6 \\
32.1 \\
32.3\end{array}$ & $\begin{array}{l}\text { Clayey silt } \\
\text { Clayey silt } \\
\text { Clayey silt } \\
\text { Clayey silt }\end{array}$ \\
\hline $\begin{array}{l}6-2(20.0) \\
6-3(20.0) \\
6-4(20.0) \\
6-5(20.0) \\
6-6(20.0)\end{array}$ & $\begin{array}{l}1.3 \\
3.4 \\
2.0 \\
2.1 \\
1.7\end{array}$ & $\begin{array}{l}62.6 \\
66.6 \\
58.7 \\
62.2 \\
60.6\end{array}$ & $\begin{array}{l}36.1 \\
30.1 \\
39.3 \\
35.7 \\
37.7\end{array}$ & $\begin{array}{l}\text { Clayey silt } \\
\text { Clayey silt } \\
\text { Clayey silt } \\
\text { Clayey silt } \\
\text { Clayey silt }\end{array}$ \\
\hline $\begin{array}{l}7-2(20.0) \\
7-3(20.0) \\
7-4(20.0) \\
7-5(20.0) \\
7-6(20.0)\end{array}$ & $\begin{array}{l}15.1 \\
17.2 \\
19.9 \\
24.7 \\
17.7\end{array}$ & $\begin{array}{l}55.8 \\
52.0 \\
56.5 \\
52.7 \\
52.4\end{array}$ & $\begin{array}{l}29.1 \\
30.8 \\
23.6 \\
22.6 \\
29.9\end{array}$ & $\begin{array}{l}\text { Clayey silt } \\
\text { Clayey silt } \\
\text { Clayey silt } \\
\text { Sand-silt-clay } \\
\text { Clayey silt }\end{array}$ \\
\hline $\begin{array}{l}8-1(20.0) \\
8-2(20.0) \\
8-3(20.0) \\
8-4(20.0) \\
8-5(20.0) \\
8-6(25.0)\end{array}$ & $\begin{array}{l}15.8 \\
15.7 \\
12.8 \\
18.7 \\
19.3 \\
15.7\end{array}$ & $\begin{array}{l}53.3 \\
52.3 \\
58.0 \\
54.9 \\
57.5 \\
56.6\end{array}$ & $\begin{array}{l}30.9 \\
32.0 \\
29.1 \\
26.5 \\
23.1 \\
27.7\end{array}$ & $\begin{array}{l}\text { Clayey silt } \\
\text { Clayey silt } \\
\text { Clayey silt } \\
\text { Clayey silt } \\
\text { Clayey silt } \\
\text { Clayey silt }\end{array}$ \\
\hline $10-1(25.0)$ & 13.6 & 55.0 & 31.5 & Clayey silt \\
\hline $\begin{array}{l}\text { DSDP 96 } \\
1-3(20.0) \\
1-4(20.0) \\
1-5(20.0) \\
1-6(20.0)\end{array}$ & $\begin{array}{l}0.0 \\
0.1 \\
0.0 \\
0.0\end{array}$ & $\begin{array}{l}18.3 \\
15.8 \\
12.4 \\
14.3\end{array}$ & $\begin{array}{l}81.7 \\
84.2 \\
87.6 \\
85.7\end{array}$ & $\begin{array}{l}\text { Clay } \\
\text { Clay } \\
\text { Clay } \\
\text { Clay }\end{array}$ \\
\hline $\begin{array}{l}2-1(20.0) \\
2-2(20.0) \\
2-2(20.0) \\
2-5(20.0) \\
2-6(20.0)\end{array}$ & $\begin{array}{r}6.3 \\
5.2 \\
10.1 \\
4.2 \\
4.6\end{array}$ & $\begin{array}{l}50.1 \\
48.2 \\
53.7 \\
50.1 \\
50.8\end{array}$ & $\begin{array}{l}43.6 \\
46.6 \\
36.2 \\
45.6 \\
44.6\end{array}$ & $\begin{array}{l}\text { Clayey silt } \\
\text { Clayey silt } \\
\text { Clayey silt } \\
\text { Clayey silt } \\
\text { Clayey silt }\end{array}$ \\
\hline $\begin{array}{l}3-2(20.0) \\
3-3(20.0) \\
3-4(20.0) \\
3-5(20.0) \\
3-6(25.0)\end{array}$ & $\begin{array}{l}5.7 \\
4.9 \\
5.9 \\
5.2 \\
4.4\end{array}$ & $\begin{array}{l}48.1 \\
45.9 \\
44.9 \\
39.3 \\
45.2\end{array}$ & $\begin{array}{l}46.1 \\
49.2 \\
49.2 \\
55.5 \\
50.3\end{array}$ & $\begin{array}{l}\text { Clayey silt } \\
\text { Silty clay } \\
\text { Silty clay } \\
\text { Silty clay } \\
\text { Silty clay }\end{array}$ \\
\hline \multicolumn{5}{|l|}{ DSDP 97} \\
\hline $\begin{array}{l}1-2(20.0) \\
1-3(20.0) \\
1-4(20.0)\end{array}$ & $\begin{array}{l}14.4 \\
10.0 \\
11.1\end{array}$ & $\begin{array}{l}31.0 \\
25.8 \\
25.1\end{array}$ & $\begin{array}{l}54.6 \\
64.2 \\
63.9\end{array}$ & $\begin{array}{l}\text { Silty clay } \\
\text { Silty clay } \\
\text { Silty clay }\end{array}$ \\
\hline $\begin{array}{l}2-2(20.0) \\
2-3(20.0) \\
2-4(20.0)\end{array}$ & $\begin{array}{l}1.2 \\
0.7 \\
1.8\end{array}$ & $\begin{array}{l}28.5 \\
30.2 \\
24.5\end{array}$ & $\begin{array}{l}70.4 \\
69.1 \\
73.7\end{array}$ & $\begin{array}{l}\text { Silty clay } \\
\text { Silty clay } \\
\text { Silty clay }\end{array}$ \\
\hline $\begin{array}{l}3-3(20.0) \\
4-3(20.0) \\
4-4(20.0) \\
4-5(20.0)\end{array}$ & $\begin{array}{l}2.1 \\
3.5 \\
8.4 \\
5.7\end{array}$ & $\begin{array}{l}35.6 \\
48.2 \\
54.7 \\
52.9\end{array}$ & $\begin{array}{l}62.2 \\
48.4 \\
36.9 \\
41.5\end{array}$ & $\begin{array}{l}\text { Silty clay } \\
\text { Silty clay } \\
\text { Clayey silt } \\
\text { Clayey silt }\end{array}$ \\
\hline $5-1(132.0)$ & 0.6 & 45.0 & 54.4 & Silty clay \\
\hline $6-1(98.0)$ & 6.6 & 31.4 & 62.0 & Silty clay \\
\hline $7-2(9.0)$ & 2.2 & 48.1 & 49.8 & Silty clay \\
\hline $\begin{array}{l}8-1(68.0) \\
8-1(95.0) \\
8-3(60.0)\end{array}$ & $\begin{array}{r}0.9 \\
4.3 \\
11.9\end{array}$ & $\begin{array}{l}13.8 \\
49.4 \\
47.4\end{array}$ & $\begin{array}{l}85.3 \\
46.3 \\
40.8\end{array}$ & $\begin{array}{l}\text { Clay } \\
\text { Clayey silt } \\
\text { Clayey silt }\end{array}$ \\
\hline
\end{tabular}




\section{ACKNOWLEDGMENTS}

These grain-size analysis were performed by D.H. Cameron, W.P. Jones, S.D. Jones, G.L. Patterson, and V.J. Sotelo. Computor processing was by P.B. Woodbury and P.L. Capps.

\section{REFERENCES}

Inman, D.I., 1952. Measures for describing the size distribution of sediment. J. Sediment. Petrol. 22, 125.
Krumbein, W.C. and Pettijohn, F.J., 1938. Manual of sedimentary petrography. New York (Appleton-Century-Crofts, Inc.), $549 \mathrm{p}$.

Shepard, F.P., 1954. Nomenclature based on sand-silt-clay ratios. J. Sediment. Petrol. 24, 151.

Wentworth, C.K., 1922. A scale of grade and class terms for clastic sediments. J. Geol. 30, 377. 